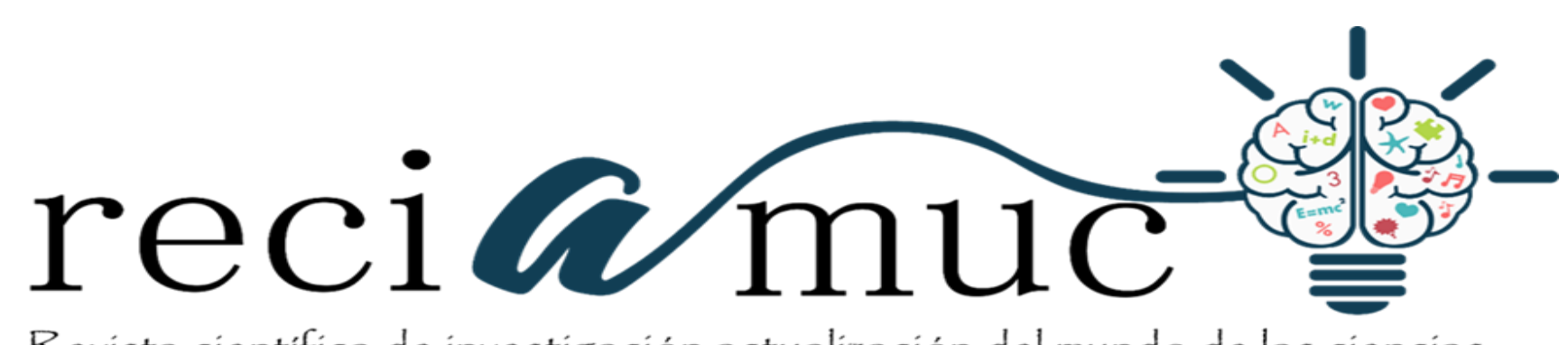

Revista cientifica de investigación actualización del mundo de las ciencias

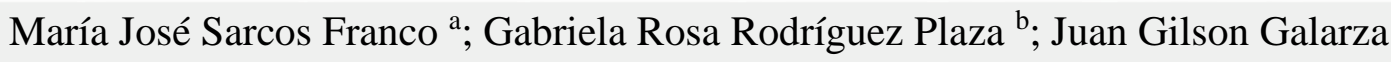
Galarza $^{c}$; Bolívar Andrés Vaca Antepara ${ }^{\mathrm{d}}$

Tratamiento y diagnóstico en profilaxis antibiótica continua con reflujos vesicoureteral

Treatment and diagnosis in continuous antibiotic prophylaxis with vesicoureteral reflux

Revista Científica de Investigación actualización del mundo de las Ciencias. Vol. 3 núm., 2, abril, ISSN: 2588-0748, 2018, pp. 417-438

DOI: $10.26820 /$ reciamuc/3.(2).abril.2019.417-438

URL: http://reciamuc.com/index.php/RECIAMUC/article/view/347

Código UNESCO: 3205 Medicina Interna

Tipo de Investigación: Artículo de Revisión

(C) RECIAMUC; Editorial Saberes del Conocimiento, 2019

Recibido: 15/01/2019

Aceptado: 07/02/2019

Publicado: 01/04/2019

Correspondencia: $\underline{\text { majo_sarcos@hotmail.com }}$

a. Médico; Investigador Independiente; Guayaquil, Ecuador;majo_sarcos@ hotmail.com

b. Médico; Investigador Independiente; Guayaquil, Ecuador;doctora.gabriela.gr@gmail.com

c. Médico; Investigador Independiente; Guayaquil, Ecuador;drjgilsongalarza@ gmail.com

d. Médico; Investigador Independiente; Guayaquil, Ecuador;bolo18_@ hotmail.com 


\section{Tratamiento y diagnóstico en profilaxis antibiótica continua con reflujos vesicoureteral}

Vol. 3, núm. 2., (2019)

María José Sarcos Franco; Gabriela Rosa Rodríguez Plaza; Juan Gilson Galarza Galarza; Bolívar Andrés Vaca Antepara

\section{RESUMEN}

El reflujo vesicoureteral (RVU) sigue siendo un problema común que se observa en pacientes pediátricos. A pesar de un gran esfuerzo de investigación, el debate sobre la manera de detectar y tratar a los pacientes es controvertida. Esta revisión pretende resumir el tratamiento del RVU con énfasis en los últimos resultados publicados en la literatura y cómo contribuyen en la mejora. Los objetivos de la gestión de RVU incluyen prevenir futuras infecciones febriles del tracto urinario, cicatrización renal, nefropatía de reflujo y la hipertensión. El enfoque de arriba hacia abajo con imagen de las vías superior y cistouretrograma miccional selectivo, es un enfoque alternativo emergente en la evaluación de niños después de su primera infección febril del tracto urinario. La eliminación de la disfunción de la vejiga y el intestino es una estrategia de gestión importante para evitar más las infecciones de este tipo, independientemente de la elección de tratamiento. La profilaxis antibiótica es una modalidad segura y eficaz para esterilizar el tracto urinario, al igual que el tratamiento endoscópico del RVU en pacientes seleccionados, aunque persisten algunas preocupaciones con respecto a su eficacia y durabilidad en comparación con el estándar de oro de ureteroneocistostomía abierta o laparoscópica. Por último, se requiere más investigación para determinar el algoritmo más eficaz y evaluar al paciente pediátrico después de la primera infección febril del tracto urinario.

Palabras clave: Profilaxis; Vejiga; Tracto urinario; Pielonefritis; Cicatrización renal; Reflujo; Reflujo vesicoureteral. 


\title{
Tratamiento y diagnóstico en profilaxis antibiótica continua con reflujos \\ vesicoureteral
}

Vol. 3, núm. 2., (2019)

María José Sarcos Franco; Gabriela Rosa Rodríguez Plaza; Juan Gilson Galarza Galarza; Bolívar Andrés Vaca Antepara

\begin{abstract}
Vesicoureteral reflux (VUR) remains a common problem seen in pediatric patients. Despite a great research effort, the debate on how to detect and treat patients is controversial. This review aims to summarize the treatment of VUR with emphasis on the latest results published in the literature and how they contribute to the improvement. The objectives of VUR management include preventing future febrile urinary tract infections, renal scarring, reflux nephropathy and hypertension. The top-down approach with image of the upper pathways and selective bladder cystourethrogram is an emerging alternative approach in the evaluation of children after their first febrile urinary tract infection. Elimination of bladder and bowel dysfunction is an important management strategy to prevent further infections of this type, regardless of the choice of treatment. Antibiotic prophylaxis is a safe and effective way to sterilize the urinary tract, as is the endoscopic treatment of VUR in selected patients, although some concerns remain regarding its efficacy and durability compared to the gold standard of open or laparoscopic ureteroneocistostomy. . Finally, more research is required to determine the most effective algorithm and evaluate the pediatric patient after the first febrile urinary tract infection.
\end{abstract}

Key words: Prophylaxis; Bladder; Urinary tract; Pyelonephritis; Renal scarring; Reflux; Vesicoureteral reflux. 


\section{Tratamiento y diagnóstico en profilaxis antibiótica continua con reflujos vesicoureteral}

Vol. 3, núm. 2., (2019)

María José Sarcos Franco; Gabriela Rosa Rodríguez Plaza; Juan Gilson Galarza Galarza; Bolívar Andrés Vaca Antepara

\section{Introducción.}

Galen, la antigua anatomista describe por primera vez el papel de la válvula de vesicoureteral en el flujo direccional de la orina en el primer siglo, sin embargo, no fue hasta que el Dr. Hutch describió una relación causal entre el reflujo vesicoureteral (RVU) y la pielonefritis en la población parapléjica que la comunidad médica de urólogos, en particular empezaron a dedicar más atención hacia la relevancia de RVU (Hutch, 1952).

Otro punto de referencia informado por los autores (Ransley \& Risdon, 1979) fortaleció el significado de RVU mediante la búsqueda de la cicatrización renal en un modelo porcino con la combinación de RVU, presiones intravesicales elevadas y orina infectada en la vejiga. En 1973, el Dr. Bailey describe "nefropatía por reflujo" como la cicatrización renal gruesa que resulta en forma de infección del tracto urinario en el ajuste del RVU (Bailey, 1973). Después de estos informes seminales sobre RVU, los estudios asociados con el RVU se han convertido en uno de los temas más investigados en toda la urología pediátrica.

La actual comprensión de la interacción entre la infección en el tracto urinario, el reflujo vesicoureteral RVU y las cicatrices renales fue fuertemente influenciada por la investigación clínica señalando la importancia y el papel de la profilaxis antibiótica en la promoción de la salud renal (Smellie, 2007). Por desgracia, a pesar de la frecuencia de RVU dentro de la población y de la extensa historia de los estudios, la evaluación y el tratamiento adecuado del paciente con RVU sigue siendo un tema polémico. 


\section{Tratamiento y diagnóstico en profilaxis antibiótica continua con reflujos \\ vesicoureteral}

Vol. 3, núm. 2., (2019)

María José Sarcos Franco; Gabriela Rosa Rodríguez Plaza; Juan Gilson Galarza Galarza; Bolívar Andrés Vaca Antepara

En Estados Unidos, tanto la Asociación Americana de Urología como la Academia Americana de Pediatría han proporcionado directrices con la esperanza de ayudar a los pediatras y a los urólogos pediátricos proporcionando evaluación para la detección y tratamiento apropiado en pacientes tanto con infecciones del tracto urinario como en presencia de RVU. En Europa, la Asociación Europea de Urología y el Instituto Nacional de Salud y Excelencia Clínica (Niza) también han tratado de establecer directrices sobre el manejo del RVU en la población pediátrica. En la disfunción de directrices, vejiga y el intestino que se ha vuelto más reconocido como un potencial determinante de futuras infecciones del tracto urinario y detección de RVU con o sin tratamiento. Este trabajo de investigación artículo no sólo relata las directrices actuales, si no que también, discute el papel creciente de disfunción directrices, vejiga y el intestino y se dirige a estudios recientes de la señal en la detección y el tratamiento del RVU.

\section{Métodos y materiales.}

Para el desarrollo de este proceso investigativo, se plantea como metodología la encaminada hacia una orientación científica particular que se encuentra determinada por la necesidad de indagar en forma precisa y coherente una situación, en tal sentido (Dávila, 2015) define la metodología "como aquellos pasos previos que son seleccionados por el investigador para lograr resultados favorables que le ayuden a plantear nuevas ideas". (p.66)

Lo citado por el autor, lleva a entender que el desarrollo de la acción investigativa busca simplemente coordinar acciones enmarcadas en una revisión bibliográfica con el fin de complementar ideas previas relacionadas Tratamiento y diagnóstico en pacientes con reflujos 


\section{Tratamiento y diagnóstico en profilaxis antibiótica continua con reflujos vesicoureteral}

Vol. 3, núm. 2., (2019)

María José Sarcos Franco; Gabriela Rosa Rodríguez Plaza; Juan Gilson Galarza Galarza; Bolívar Andrés Vaca Antepara

vesicoureteral, a través, de una revisión de literatura, para así finalmente elaborar un cuerpo de consideraciones generales que ayuden a ampliar el interés propuesto.

\section{Tipo de Investigación.}

Dentro de toda práctica investigativa, se precisan acciones de carácter metodológico mediante las cuales, se logra conocer y proyectar los eventos posibles que la determinan, así como las características que hacen del acto científico un proceso interactivo ajustado a una realidad posible de ser interpretada. En este sentido, se puede decir, que la presente investigación corresponde al tipo documental, definido por Castro (2016), "se ocupa del estudio de problemas planteados a nivel teórico, la información requerida para abordarlos se encuentra básicamente en materiales impresos, audiovisuales y /o electrónicos". (p.41).

En consideración a esta definición, la orientación metodológica permitió la oportunidad de cumplir con una serie de actividades inherentes a la revisión y lectura de diversos documentos donde se encontraron ideas explicitas relacionadas con los tópicos encargados de identificar a cada característica insertada en el estudio. Por lo tanto, se realizaron continuas interpretaciones con el claro propósito de revisar aquellas apreciaciones o investigaciones propuestas por diferentes investigadores relacionadas con el tema de interés, para luego dar la respectiva argumentación a los planteamientos, en función a las necesidades encontradas en la indagación. 


\section{Tratamiento y diagnóstico en profilaxis antibiótica continua con reflujos}

vesicoureteral

Vol. 3, núm. 2., (2019)

María José Sarcos Franco; Gabriela Rosa Rodríguez Plaza; Juan Gilson Galarza Galarza; Bolívar

Andrés Vaca Antepara

\section{Fuentes Documentales.}

El análisis correspondiente a las características que predomina en el tema seleccionado, llevan a incluir diferentes fuentes documentales encargadas de darle el respectivo apoyo y en ese sentido cumplir con la valoración de los hechos a fin de generar nuevos criterios que sirven de referencia a otros procesos investigativos. Para (CASTRO, 2016) las fuentes documentales incorporadas en la investigación documental o bibliográfica, "representa la suma de materiales sistemáticos que son revisados en forma rigurosa y profunda para llegar a un análisis del fenómeno".(p.41). Por lo tanto, se procedió a cumplir con la realización de una lectura previa determinada para encontrar aquellos aspectos estrechamente vinculados con el tema, con el fin de explicar mediante un desarrollo las respectivas apreciaciones generales de importancia.

\section{Técnicas para la recolección de la información.}

La conducción de la investigación para ser realizada en función a las particularidades que determinan a los estudios documentales, tiene como fin el desarrollo de un conjunto de acciones encargadas de llevar a la selección de técnicas estrechamente vinculadas con las características del estudio. En tal sentido, (Bolívar, 2015), refiere, que es "una técnica particular para aportar ayuda a los procedimientos de selección de las ideas primarias y secundarias”. (p. 71).

Por ello, se procedió a la utilización del subrayado, resúmenes, fichaje, como parte básica para la revisión y selección de los documentos que presentan el contenido teórico. Es decir, que mediante la aplicación de estas técnicas se pudo llegar a recoger informaciones en cuanto a la revisión bibliográfica de los diversos elementos encargados de orientar el proceso de investigación. 


\section{Tratamiento y diagnóstico en profilaxis antibiótica continua con reflujos vesicoureteral}

Vol. 3, núm. 2., (2019)

María José Sarcos Franco; Gabriela Rosa Rodríguez Plaza; Juan Gilson Galarza Galarza; Bolívar Andrés Vaca Antepara

Tal como lo expresa, (Bolívar, 2015) "las técnicas documentales proporcionan las herramientas esenciales y determinantes para responder a los objetivos formulados y llegar a resultados efectivos" (p. 58). Es decir, para responder con eficiencia a las necesidades investigativas, se introdujeron como técnica de recolección el método inductivo, que hizo posible llevar a cabo una valoración de los hechos de forma particular para llegar a la explicación desde una visión general.

Asimismo, se emplearon las técnicas de análisis de información para la realización de la investigación que fue ejecutada bajo la dinámica de aplicar diversos elementos encargados de determinar el camino a recorrer por el estudio, según, (Bolívar, 2015) las técnicas de procesamiento de datos en los estudios documentales "son las encargadas de ofrecer al investigador la visión o pasos que debe cumplir durante su ejercicio, cada una de ellas debe estar en correspondencia con el nivel a emplear" (p. 123). Esto indica, que para llevar a cabo el procesamiento de los datos obtenidos una vez aplicado las técnicas seleccionadas, tales como: fichas de resumen, textual, registros descriptivos entre otros, los mismos se deben ajustar al nivel que ha sido seleccionado.

\section{Resultados.}

Etiología

El flujo de la orina desde el uréter a la vejiga es normalmente direccional a través de la unión vesicoureteral, normalmente el RVU no se produce porque no hay compresión del túnel, el uréter es la submucosa medida de la vejiga que se llena de orina. El RVU es una anomalía del 


\section{Tratamiento y diagnóstico en profilaxis antibiótica continua con reflujos \\ vesicoureteral}

Vol. 3, núm. 2., (2019)

María José Sarcos Franco; Gabriela Rosa Rodríguez Plaza; Juan Gilson Galarza Galarza; Bolívar Andrés Vaca Antepara

desarrollo creado por una longitud inadecuada del túnel submucoso intravesical del uréter en relación a las presiones experimentadas por la vejiga del paciente (Fig. $\left.\mathrm{N}^{\circ} 1\right)$.

Figura $N^{0} 1$. Anatomía de la unión del ureterovesical.

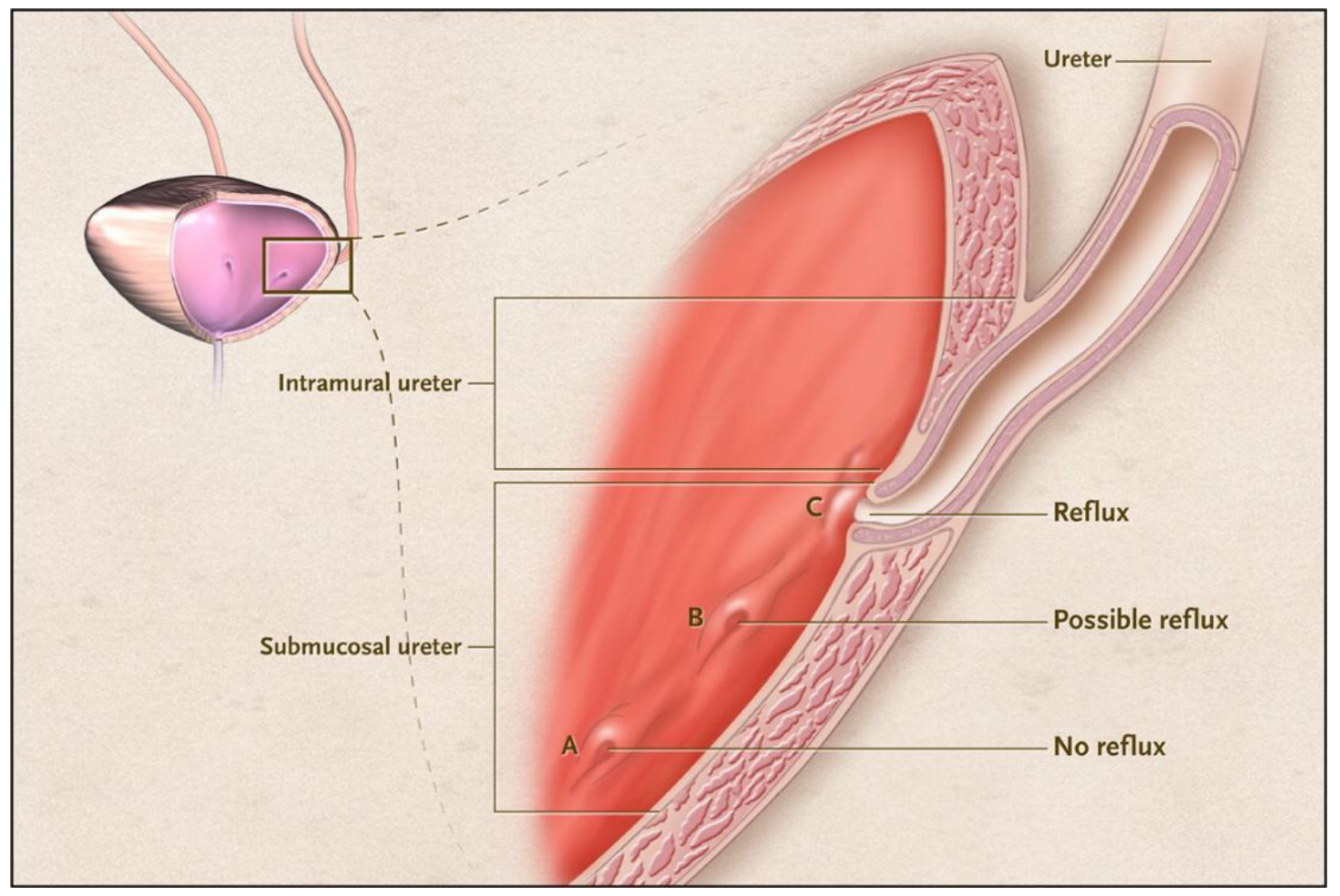

Fuente: (Diamond \& Mattoo, 2012).

Hoy en día el RVU se clasifica como una patología primaria o secundaria. Se considera primaria si se piensa que es una deficiencia aislada en el túnel ureteral anatómico. Las dimensiones anatómica y fisiológica del túnel normal son de $5 \mathrm{~cm}$ longitud a $1 \mathrm{~cm}$ diámetro. El RVU secundaria se refiere a que es debido a las dinámicas anormales de la vejiga y / o presiones de micción elevadas. Estas anomalías se ven con disfunción del tracto urinario inferior derivada de obstrucción de la salida de la vejiga (válvulas uretrales posteriores o ureterocele), vejiga neurogénica, o micción disfuncional. En RVU secundario, el túnel vesicoureteral puede o no ser de longitud 


\section{Tratamiento y diagnóstico en profilaxis antibiótica continua con reflujos vesicoureteral}

Vol. 3, núm. 2., (2019)

María José Sarcos Franco; Gabriela Rosa Rodríguez Plaza; Juan Gilson Galarza Galarza; Bolívar Andrés Vaca Antepara

normal, sin embargo, debido a las presiones de micción intravesicales elevadas, el túnel vesicoureteral es "insuficiente" para evitar RVU. La literatura antigua no apreciaba esta distinción del RVU y era común ver los informes de los pacientes con reflujo primario con predominio de la disfunción de la vejiga. (Taylor, 2012)

\section{Epidemiología}

Hay una relación familiar entre la existencia de RVU en pacientes, aproximadamente el $30 \%$ de los hermanos de los pacientes diagnosticados con RVU presentan antecedentes de padecer de acuerdo a estudios de cribado. El RVU primario se encuentra con una prevalencia significativamente mayor en los familiares cercanos a aquellos con reflujo. Los autores (Kaefer, Curran, \& T, 2000) encontraron que la prevalencia de RVU fue del $80 \%$ entre gemelos idénticos y que la prevalencia era del $35 \%$ en los gemelos no idénticos o mellizos.

En un estudio realizado por (Scott, Swallow, \& Coulthard, 2010) durante un período de 3 años acerca del RVU familiar en pacientes que acudieron a las consultas de atención prenatal, con recién nacidos de padres u otros miembros de la familia con diagnóstico definitivo de cistografía o probable RVU se obtuvo que 211 con antecedentes familiares confirmado, tuvieron una prevalencia de RVU en el $31 \%$ de los pacientes.

- Proyección de hermanos

Hoy en día, el cribado de rutina para los hermanos con RVU ya no se practica. Para aquellos con un hermano o hermana con RVU, las directricesde la Asociación Americana de Urología 


\section{Tratamiento y diagnóstico en profilaxis antibiótica continua con reflujos}

vesicoureteral

Vol. 3, núm. 2., (2019)

María José Sarcos Franco; Gabriela Rosa Rodríguez Plaza; Juan Gilson Galarza Galarza; Bolívar Andrés Vaca Antepara

sugeriría consideración para una cistouretrografía miccional (CUGM) sólo si hay evidencia de daño renal en las imágenes o antecedentes de una infección urinaria significativa (Peters, Skoog, \& Arant, 2010). Del mismo modo, las directrices de la Asociación Europea de Urología sugieren una CUGM si hay evidencia del daño renal en la ecografía o un historial de infección del tracto urinario. En niños mayores y asintomáticos, no se recomienda la detección de RVU. En general, la detección de hermanos ha disminuido en la práctica como se evalúa de forma selectiva los niños en base a un perfil de estratificación del riesgo en lugar de la pantalla de manera uniforme todos los hermanos.

\section{Diagnóstico de RVU}

Tradicionalmente, un episodio agudo de pielonefritis provocó un trabajo de diagnóstico que incluía un CUGM, y esto sigue siendo la prueba de imagen estándar de oro para el diagnóstico de RVU. El fundamento de las pruebas CUGM en el entorno de la pielonefritis se basa en la mayor prevalencia de RVU se diagnostica después de una infección urinaria significativa a menudo con un cuadro febril. Un metaanálisis realizado por (Faust, 2009) determinó que el RVU puede ser el principal factor de riesgo que predisponente a niños a la cicatrización cortical renal adquirida aproximadamente, posiblemente por el aumento del riesgo de infecciones urinarias recurrentes.

Con la adopción generalizada de la ecografía prenatal para el cuidado materno-fetal, ahora otra población objetivo para la evaluación diagnóstica incluye aquellos bebés con anormalidades persistentes en una ecografía posnatal. En estos pacientes, la Asociación Americana de Urología recomienda CUGM para aquellos con hidronefrosis de grado 3 o 4, hidrouréter o una ecografía 


\section{Tratamiento y diagnóstico en profilaxis antibiótica continua con reflujos vesicoureteral}

Vol. 3, núm. 2., (2019)

María José Sarcos Franco; Gabriela Rosa Rodríguez Plaza; Juan Gilson Galarza Galarza; Bolívar Andrés Vaca Antepara

anormal de la vejiga prenatal a ambos tardía o ecografía posnatal. Los pacientes con menor grado de hidronefrosis se dan las opciones de CUGM u observación (Peters, 2010). La Asociación Europea de Urología reconoce que el grado de hidronefrosis no es un indicador fiable de RVU. Sin embargo, CUGM se recomienda para las personas con alto grado hidronefrosis bilateral, riñones dúplex con hidronefrosi, ureterocele, dilatación ureteral o vejigas que aparecen anormales.

Una vez que se estableció el diagnóstico de RVU en CUGM, el RVU se clasifica o es clasificado en gravedad por el grado de dilatación ureteral en CUGM. El sistema de clasificación para el RVU mundialmente aceptado se basa en el sistema de clasificación radiológica establecido por el Comité de Estudios de reflujo Internacional en 1981 (Lebowitz, Olbing, \& Parkkulainen, 1985). El grado I muestra reflujo en un uréter no dilatado; el grado II, en la pelvis y los cálices sin dilatación; el grado III, de leve a moderada dilatación del uréter, pelvis renal y los cálices con embotamiento mínimo de fondos de saco; el grado IV tortuosidad moderada ureteral y la dilatación de la pelvis y los cálices y el grado $\mathrm{V}$, dilatación bruta de uréter, la pelvis y los cálices, pérdida de impresiones papilares y tortuosidad ureteral.

Protocolos de detección y diagnóstico

El reto en la evaluación y manejo del RVU se debe principalmente a la falta de consenso de los pacientes y qué pacientes requieren tratamiento. Como se describió anteriormente, la mayoría de los médicos utilizan la ecografía renal y vesical como un barómetro para decidir quién debe tener una CUGM. La ecografía renal y vesical es una herramienta de cribado ideal, ya que es barato, no invasivo y no utiliza radiación. Sin embargo, desafortunadamente algunos estudios han 


\section{Tratamiento y diagnóstico en profilaxis antibiótica continua con reflujos}

vesicoureteral

Vol. 3, núm. 2., (2019)

María José Sarcos Franco; Gabriela Rosa Rodríguez Plaza; Juan Gilson Galarza Galarza; Bolívar Andrés Vaca Antepara

encontrado que se ven obstaculizados por la mala sensibilidad y valor predictivo negativo para la determinación de RVU (Massanyi, Preece, \& Gupta, 2013). En un estudio comparativo de ultrasonidos y formación de imágenes CUGM dentro de una población de recién nacidos con una historia familiar de RVU, hubo una baja sensibilidad y un valor predictivo pobre para detectar RVU al evaluar prenatal y diámetros de la pelvis renal postnatal. Las limitaciones de RBUS están resaltados por los informes de resultados ecografía renal y vesical normales en hasta un $60 \%$ de los casos de RVU a pesar de haberse perdido $50 \%$ de las anomalías renales observadas en la gammagrafía dimercaptosuccínico ácido renal (MacKenzie, Fowler, \& Hollman, 2014). Los inconvenientes de las pruebas CUGM son la naturaleza invasiva, el potencial de exposición a la radiación y el riesgo de potencial de infección del tracto urinario iatrogénica con uretral y la cateterización de la vejiga. La preocupación de radiación se ha disminuido con películas de dosimetría y limitado punto y la naturaleza invasiva ha sido templada con sedación. Sin embargo, en comparación con CUGM, la ecografía renal y vesical claramente provoca más ansiedad para los pacientes y las familias, debido al potencial de exposición a la radiación y el riesgo de infección del tracto urinario iatrogénica con uretral y la cateterización de la vejiga.

- Enfoque de arriba hacia abajo

Como una alternativa al enfoque tradicional de prueba CUGM temprano en la evaluación diagnóstica, algunos médicos promueven un enfoque "de arriba abajo". La razón fundamental para el enfoque de arriba hacia abajo es que sólo el RVU que se traduce en la pielonefritis es clínicamente relevante. Con el conocimiento de que sólo pielonefritis conduce a la cicatrización renal, este método emplea una exploración renal con ácido dimercaptosuccínico pronto después 


\section{Tratamiento y diagnóstico en profilaxis antibiótica continua con reflujos vesicoureteral}

Vol. 3, núm. 2., (2019)

María José Sarcos Franco; Gabriela Rosa Rodríguez Plaza; Juan Gilson Galarza Galarza; Bolívar Andrés Vaca Antepara

de un confirmado la infección del tracto urinario febril. Aquellos con un examen negativo no requerirían ninguna evaluación adicional a menos que se observe infección urinaria recurrente. Sin embargo, el CUGM se llevaría a cabo sobre los que tienen confirmada, fotopenia focal sobre la exploración renal con ácido dimercaptosuccínico.

En una evaluación retrospectiva del enfoque de arriba hacia abajo, realizada por el autor (Hansson, 2004) a 303 niños menores de 2 años de edad que tenían una exploración renal con ácido dimercaptosuccínico y CUGM se lleva a cabo después de su primera Infección del tracto urinario. A pesar de una tasa de $82 \%$ de infección febril del tracto urinario, RVU se encontró en sólo el $26 \%$ de los que tienen infección febril del tracto urinario y RVU, el $66 \%$ tenía una exploración renal con ácido dimercaptosuccínico anormal. En esta cohorte de pacientes con exploración renal con ácido dimercaptosuccínico anormal, el enfoque de arriba hacia abajo habría identificado solamente el 66\% de aquellos con RVU y el riesgo de futuras cicatrices y excluidos del 40\% de la cohorte sin RVU de un CUGM. De los siete pacientes con dilatación, grado III RVU y una exploración renal con ácido dimercaptosuccínico normal, todos excepto uno tenían una infección febril del tracto urinaria inicial. Además, estos mismos 7 pacientes con exploración renal con ácido dimercaptosuccínico normales se encontraron en el seguimiento de tener una resolución completa de RVU (5 pacientes) o degradados a vesicoureteral grado I (2 pacientes).

La ventaja del enfoque de abajo superior es que los pacientes con resultados anormales en la exploración renal con ácido dimercaptosuccínico serían evaluados de forma selectiva, ya que la mayoría de los pacientes con RVU e infección del tracto urinario no desarrollan daño renal. Al dirigirse a una cohorte en el riesgo de sufrir un CUGM, un segmento de niños con infección febril 


\section{Tratamiento y diagnóstico en profilaxis antibiótica continua con reflujos}

vesicoureteral

Vol. 3, núm. 2., (2019)

María José Sarcos Franco; Gabriela Rosa Rodríguez Plaza; Juan Gilson Galarza Galarza; Bolívar Andrés Vaca Antepara

del tracto urinario estaría protegido de la morbilidad de hacer más pruebas y el tratamiento potencial. Desafortunadamente, el uso creciente de la exploración renal con ácido dimercaptosuccínico tiene limitaciones. Estas limitaciones incluyen la poca disponibilidad del isótopo para la exploración renal con ácido dimercaptosuccínico, el requisito de una línea intravenosa, los requisitos de sedación frecuentes, pequeña exposición a la radiación, y mayores costos financieros. Por último, la mayoría de los pacientes encontraron que la cicatrización renal con exploración renal con ácido dimercaptosuccínico anormales tienen una CUGM negativa y la persistencia de las lesiones del parénquima renal es independiente del RVU. (Ditchfield, 2004)

Curiosamente, la exploración renal con ácido dimercaptosuccínico con anormalidades en conjunción con alto grado de RVU (por ejemplo, los grados 3- 5) están asociados con una mayor posibilidad de avance infección del tracto urinario (60\%) a pesar de la profilaxis con antibióticos en comparación con aquellos con una exploración renal con ácido dimercaptosuccínico normal y un grado de RVU similar (8\%) (Niguyen, 2004). Además, sólo el 5\% de los que tienen una exploración renal con ácido dimercaptosuccínico anormal mostraron mejoría de RVU comparado con el 46\% que se resolvió en el grupo de exploración renal con ácido dimercaptosuccínico normal. Al hacer referencia a las directrices Asociación Americana de Urología, imágenes de exploración renal con ácido dimercaptosuccínico se recomienda en la fijación de un ultrasonido renal anormal que sugiere cicatrización o un suero queratinizan elevada [12]. Las Directrices de la Asociación Europea de Urología mencionar el enfoque de arriba hacia abajo alternativa y describir el potencial de perder 5-27\% de los casos de RVU, con significación dudosa [13]. 


\section{Tratamiento y diagnóstico en profilaxis antibiótica continua con reflujos vesicoureteral}

Vol. 3, núm. 2., (2019)

María José Sarcos Franco; Gabriela Rosa Rodríguez Plaza; Juan Gilson Galarza Galarza; Bolívar Andrés Vaca Antepara

Opciones de tratamiento

- Profilaxis antibiótica continua

Durante años, la eficacia de la profilaxis antibiótica continua ha sido cuestionada debido a sus potenciales riesgos tales como selección organismos resistentes, síndrome de Stevens-Johnson entre otros, además de que beneficios inciertos (Pennesi \& Peratoner, 2008). Estudios posteriores proporcionan una clara evidencia de la eficacia de la profilaxis antibiótica continua, en comparación con la observación. Los ejemplos más notables descritos por el autor (Craig, 2009) incluyen en su estudio PRIVENT y el ensayo de reflujo en niños. Estos estudios, sin embargo, demostraron un pequeño beneficio de la profilaxis antibiótica continua. Algunos argumentaron que los resultados fueron estadísticamente, pero no clínicamente significativa. Otros argumentaron que estos estudios sólo incluyeron un subconjunto de pacientes con RVU de alto grado, y por lo tanto no eran generalizables a la población entera RVU.

En las Guías Clínicas Asociación Americana de Urología, el beneficio de la profilaxis antibiótica continua se basa en el uso recomendado de criterios de selección para determinar un perfil de riesgo de futuras infecciones urinarias (Peters, 2010). Los grupos de alto riesgo que se beneficiarían de la profilaxis antibiótica continua incluyen aquellos $<1$ año de edad, dilatando RVU grados 3-5 y / o antecedentes de infección del tracto urinario febril. Para aquellos > 1 año de edad, la profilaxis antibiótica continua se recomienda cuando existe disfunción de la vejiga y el intestino. De lo contrario, la profilaxis antibiótica continua se considera opcional para aquellos $>1$ año de edad. Las directrices de la Asociación Europea de Urología sugieren que, 


\section{Tratamiento y diagnóstico en profilaxis antibiótica continua con reflujos}

vesicoureteral

Vol. 3, núm. 2., (2019)

María José Sarcos Franco; Gabriela Rosa Rodríguez Plaza; Juan Gilson Galarza Galarza; Bolívar Andrés Vaca Antepara

independientemente del grado de reflujo o presencia de cicatrices renales, todos los pacientes diagnosticados a la edad de $<1$ año pueden ser tratados inicialmente con profilaxis antibiótica continua. La corrección quirúrgica o endoscópica definitiva se prefiere para las personas con infecciones intercurrentes frecuentes. En todos los niños que se presentan a la edad de 1-5 años, la profilaxis antibiótica continua es la opción preferida, inicial.

\section{- Cirugía}

La recomendación para el tratamiento quirúrgico es un proceso de toma de decisiones compartida que implica a los padres, tutores y el médico. La decisión está influenciada por múltiples factores, incluyendo el riesgo-beneficio de la intervención, la presencia de avance de infecciones del tracto urinario y también la ansiedad de las pruebas CUGM con RVU persistente.

El tratamiento endoscópico es un paciente ambulatorio, es una opción atractiva, mínimamente invasivo en el tratamiento de RVU.

El tratamiento endoscópico ha reaparecido en el tratamiento quirúrgico para el RVU desde que la Administración Federal de Medicamentos aprobó dextranómero copolímero de ácido hialurónico [Deflux ${ }^{\circledR}$ ] en 2001 para la corrección endoscópica de grados II-IV RVU (Nelson \& Copp, 2009). La tasa de éxito del tratamiento endoscópico es de aproximadamente 60-90\% y es más dependiente e influenciado por el grado de RVU y la ausencia de disfunción de la vejiga y el intestino. La modificación de las técnicas endoscópicas mediante el uso de múltiples puntos de inyección submucosa en el orificio uretral se ha reportado para mejorar las tasas de éxito. Un metaanálisis realizado por el autor (Elder, 2006), sugiere que el factor pronóstico más importante 


\section{Tratamiento y diagnóstico en profilaxis antibiótica continua con reflujos vesicoureteral}

Vol. 3, núm. 2., (2019)

María José Sarcos Franco; Gabriela Rosa Rodríguez Plaza; Juan Gilson Galarza Galarza; Bolívar Andrés Vaca Antepara

en el éxito del tratamiento endoscópico es el grado de RVU pre-operatorio y no la técnica en particular.

La técnica de abrir neoureterocistotomía o de reimplantación ureteral es el "patrón oro" para la corrección quirúrgica del RVU. La tasa de éxito de la reimplantación ureteral es de aproximadamente $95 \%$, independientemente del grado de RVU o presencia de disfunción de la vejiga y el intestino. La reimplantación ureteral corrige una longitud túnel ureteral inadecuada mediante el establecimiento de un mecanismo valvular que permite la compresión pasiva del uréter submucoso durante el llenado de la vejiga. La clave de característica quirúrgica en la construcción del uréter reimplantado está estableciendo una relación 5: 1 en el diámetro de túnel de longitud a ureteral . Hay múltiples variaciones quirúrgicas de reimplantación ureteral abierto que implican intravesical, extravesicales disecciones intra y extravesicales o combinados de. (Marshall, 2015)

La cruz-trigonal, reimplante ureteral de Cohen es una movilización del uréter que mantiene el hiato de inserción original en la pared de la vejiga (Cohen, 2007). A diferencia de la técnica de Glenn-Anderson, el uréter se reimplantado en todo el trígono hacia el uréter contralateral. Este técnico quirúrgico es particularmente ventajoso en la creación de un túnel largo para uréteres dilatados reimplantados. Algunos sostienen, sin embargo, que esta técnica afecta a la anatomía por lo que el acceso endoscópico a los uréteres más difíciles es necesario en el futuro (por ejemplo, para la urolitiasis), pero esto no parece ser un problema en sentido longitudinal.

Las Asociación Americana de Urología considera una intervención quirúrgica como una “opción” en el manejo del RVU (Peters, Skoog, \& Arant, 2010). En un estudio Internacional de 


\section{Tratamiento y diagnóstico en profilaxis antibiótica continua con reflujos}

vesicoureteral

Vol. 3, núm. 2., (2019)

María José Sarcos Franco; Gabriela Rosa Rodríguez Plaza; Juan Gilson Galarza Galarza; Bolívar Andrés Vaca Antepara

reflujo en niños en comparación con los niños <11 años de edad con RVU asignados aleatoriamente a una intervención quirúrgica o profilaxis antibiótica continua, no mostró diferencias en las cicatrices renales "urográficos", la tasa de recurrencia de infección del tracto urinario, o el crecimiento renal. Sin embargo, hubo un aumento en las infecciones del tracto urinario febril en el grupo profilaxis antibiótica continúa encontrado después de diez años de seguimiento (Jodal, Smellie, \& Lax, 2013). Posteriormente, recomiendan que pacientes que reciben profilaxis antibiótica continua con un cuadro febril de avance de infección del tracto urinario deben ser considerados para la reimplantación quirúrgica abierta ureteral o inyección endoscópica, con intención curativa.

\section{Conclusión.}

La evaluación y el tratamiento del RVU ha cambiado significativamente en las últimas dos décadas. El aumento del conocimiento de la historia natural de RVU y la identificación de grupos de alto riesgo ha adaptado la realización de decisiones clínicas y pruebas de RVU. La imagen exploración renal con ácido dimercaptosuccínico ahora se utilizan con frecuencia en lugar de un CUGM inmediato. Sabemos que las infecciones urinarias son un factor determinante importante en el tratamiento del RVU y que el control de infecciones para la infección urinaria deja sin efecto la importancia de RVU. Otro concepto importante que se entiende mejor en el manejo del RVU es el papel de disfunción de la vejiga y el intestino. La disfunción de la vejiga y el intestino son un factor de riesgo significativo para la prevalencia de las infecciones urinarias y el desarrollo del RVU secundario. La evaluación de disfunción de la vejiga y el intestino en pacientes esfínteres 


\section{Tratamiento y diagnóstico en profilaxis antibiótica continua con reflujos vesicoureteral}

Vol. 3, núm. 2., (2019)

María José Sarcos Franco; Gabriela Rosa Rodríguez Plaza; Juan Gilson Galarza Galarza; Bolívar Andrés Vaca Antepara

con RVU y las infecciones urinarias se debe realizar de forma rutinaria, como la gestión exitosa de disfunción de la vejiga y el intestino promueve la degradación o eliminación de RVU.

Nuevos hallazgos muestran que la profilaxis antibiótica continua se asocia con un riesgo sustancialmente reducido de recurrencia de infección del tracto urinario en niños con RVU, pero no de cicatrización renal. Por último, la corrección quirúrgica del RVU en pacientes seleccionados sigue siendo una opción de tratamiento definitiva y existe una amplia gama de técnicas quirúrgicas que pueden adaptarse para el tratamiento del RVU.

\section{Bibliografía.}

Bailey, R. (1973). The relationship of vesicoureteric reflux to urinary tract infection and chronic pyelonephritis-reflux nephropathy. Clin Nephrol.

Bolívar, J. (2015). Investigación Documental. México. Pax.

Castro, J. (2016). Técnicas Documentales. México. Limusa.

Cohen. (2007). La técnica de reimplantación de Cohen. Defectos de nacimiento. Orig Artic.

Craig, J. (2009). Prevention of recurrent urinary tract infection in children with vesicoureteric reflux and normal renal tracts [PRIVENT] investigators. Antibiotic prophylaxis and recurrent urinary tract infection in children . Engl J Med , 48-59.

Davila, A. (2015). Diccionario de Términos Científicos. . Caracas: Editorial Oasis.

Diamond, D., \& Mattoo, T. (2012). . tratamiento endoscópico del reflujo vesicoureteral primario. $18-26$.

Ditchfield, R. (2004). Persistent renal cortical scintigram defects in children 2 years after urinary tract infection. Pediatr Radiol

Elder, S. (2006). Endoscopic therapy for vesicoureteral reflux: a meta-analysis. I reflux resolution and urinary tract infection. 


\section{Tratamiento y diagnóstico en profilaxis antibiótica continua con reflujos}

vesicoureteral

Vol. 3, núm. 2., (2019)

María José Sarcos Franco; Gabriela Rosa Rodríguez Plaza; Juan Gilson Galarza Galarza; Bolívar

Andrés Vaca Antepara

Faust, W. (2009). Incidence of post-pyelonephritic renal scarring: a meta-analysis of the dimercapto-succinic acid literature. (págs. 290-7.). J Urol.

Hansson, S. (2004). Gammagrafía con ácido dimercapto-succínico en lugar de anular la cistouretrografía para lactantes con infección del tracto urinario. 71-3.

Hutch. (1952). Vesicoureteral reflux in the paraplegic: cause and correction. . J Urol .

Jodal, Smellie, \& Lax. (2013). Resultados a diez años del tratamiento aleatorizado de niños con reflujo vesicoureteral severo. Informe final del Estudio Internacional de Reflujo en Niños. . Pediatr Nephrol.

Kaefer, M., Curran, M., \& T, T. (2000). Sibling vesicoureteral reflux in multiple gestation births. Pediatrics , 800-4.

Lebowitz, Olbing, \& Parkkulainen. (1985). International system of radiographic grading of vesicoureteric reflux. . (págs. 105-9.). Pediatr Radiol .

MacKenzie, Fowler, \& Hollman. (2014). El valor del ultrasonido en el niño con una infección aguda del tracto urinario. 240-4.

Marshall. (2015). Ureterovesicoplastia: selección de pacientes, incidencia y evitación de complicaciones. Una revisión de 3527 casos.

Massanyi, Preece, \& Gupta. (2013). Utilidad de la ecografía de detección después de la primera infección urinaria febril entre pacientes con reflujo vesicoureteral clínicamente significativo. 05-9.

Nelson, \& Copp. (2009). Is availability of endoscopy changing initial management of vesicoureteral reflux? 189-245.

Niguyen, T. (2004). Abnormal dimercapto-succinic acid scans predict an increased risk of breakthrough infection in children with vesicoureteral reflux.

Pennesi, \& Peratoner. (2008). ¿La profilaxis antibiótica en niños con reflujo vesicoureteral es efectiva para prevenir la pielonefritis y las cicatrices renales? Un ensayo aleatorizado y controlado. Pediatría.

Peters. (2010). Summary of the AUA guideline on management of primary vesicoureteral reflux in children. (págs. 34-44.). J Urol.

Peters, C., Skoog, S., \& Arant, B. (2010). Resumen de la guía de la AUA sobre el tratamiento del reflujo vesicoureteral primario en niños. Mexico: J Urol. 
Tratamiento y diagnóstico en profilaxis antibiótica continua con reflujos vesicoureteral

Vol. 3, núm. 2., (2019)

María José Sarcos Franco; Gabriela Rosa Rodríguez Plaza; Juan Gilson Galarza Galarza; Bolívar Andrés Vaca Antepara

Ransley, \& Risdon. (1979). The pathogenesis of reflux nephropathy. Contrib Nephrol.

Scott, E., Swallow, V., \& Coulthard, G. (2010). Screening of newborn babies for familial ureteric reflux. Lancet.

Smellie, J. (2007). Reflections on 30 years of treating children with urinary tract infections. .

Taylor, A. (2012). Quantitation of renal function with static imaging agents. Semin Nucl Med, (págs. 330-44.).

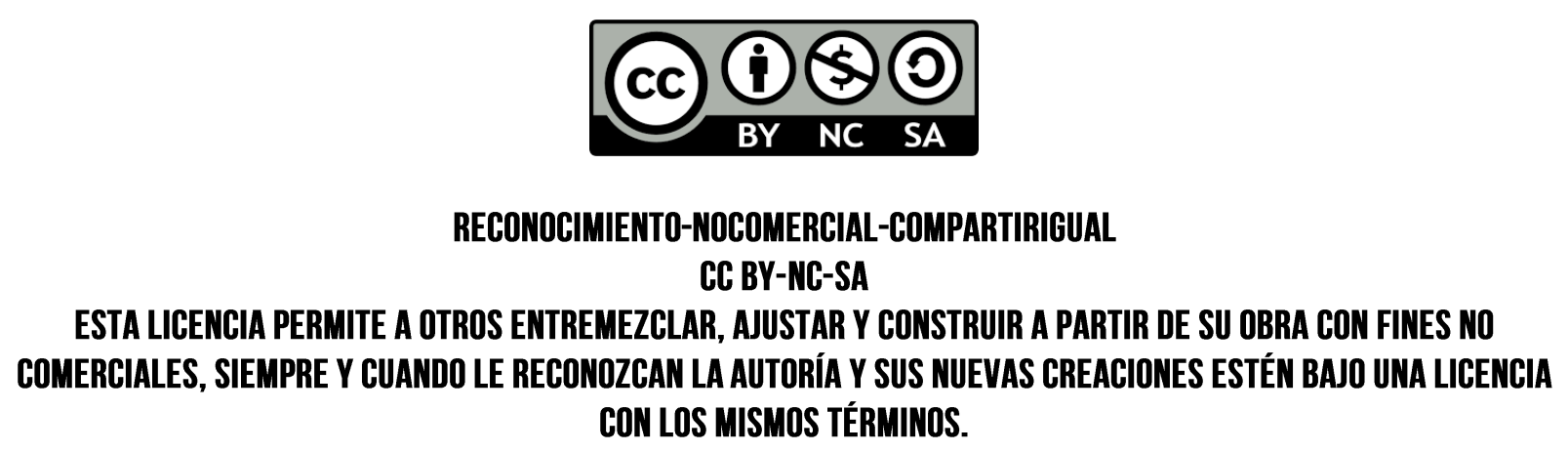

